\title{
Phytochemical Flavone Confers Broad-Spectrum Tolerance to Insecticides in Spodoptera litura by Activating ROS/CncC- Mediated Xenobiotic Detoxification Pathways
}

Kai Lu', Yibei Cheng1, Yimin Li ${ }^{1}$, Wenru Li $^{1}$, Rensen Zeng ${ }^{2}$, Yuanyuan Song ${ }^{2 *}$

${ }^{1}$ State Key Laboratory of Ecological Pest Control for Fujian and Taiwan Crops, College of Life Sciences, Fujian Agriculture and Forestry University, Fuzhou, 350002, China

${ }^{2}$ Key Laboratory of Ministry of Education for Genetics, Breeding and Multiple Utilization of Crops, College of Agriculture, Fujian Agriculture and Forestry University, Fuzhou, 350002, China

\section{* Corresponding Author}

Prof. Yuanyuan Song

E-mail address: yyuansong@,fafu.edu.cn

Tel.: +86-0591-83789272.

Running head: Flavone enhances insecticide tolerance 


\section{Supporting Information}

Fig. $\mathbf{S 1}$
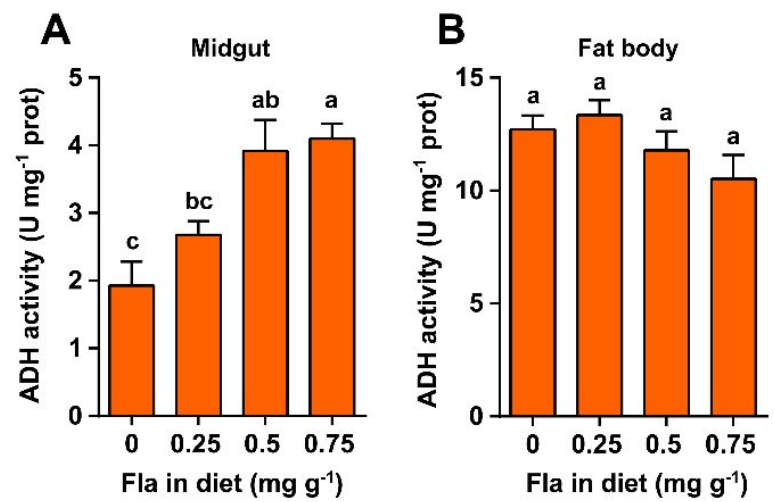

Figure S1. Activities of alcohol dehydrogenase (ADH) of flavone-treated S. litura larvae. The $4^{\text {th }}$ instar larvae were fed on artificial diet containing different doses of flavone for $48 \mathrm{~h}$; then, the midguts (A) and fat bodies (B) were collected for enzymatic analyses. Results are expressed as means \pm SE of four biological replicates. Different lowercase letters above the bars indicate significant differences (one-way ANOVA followed by Tukey HSD multiple comparison test, $P<0.05$ ). 


\section{Fig. S2}
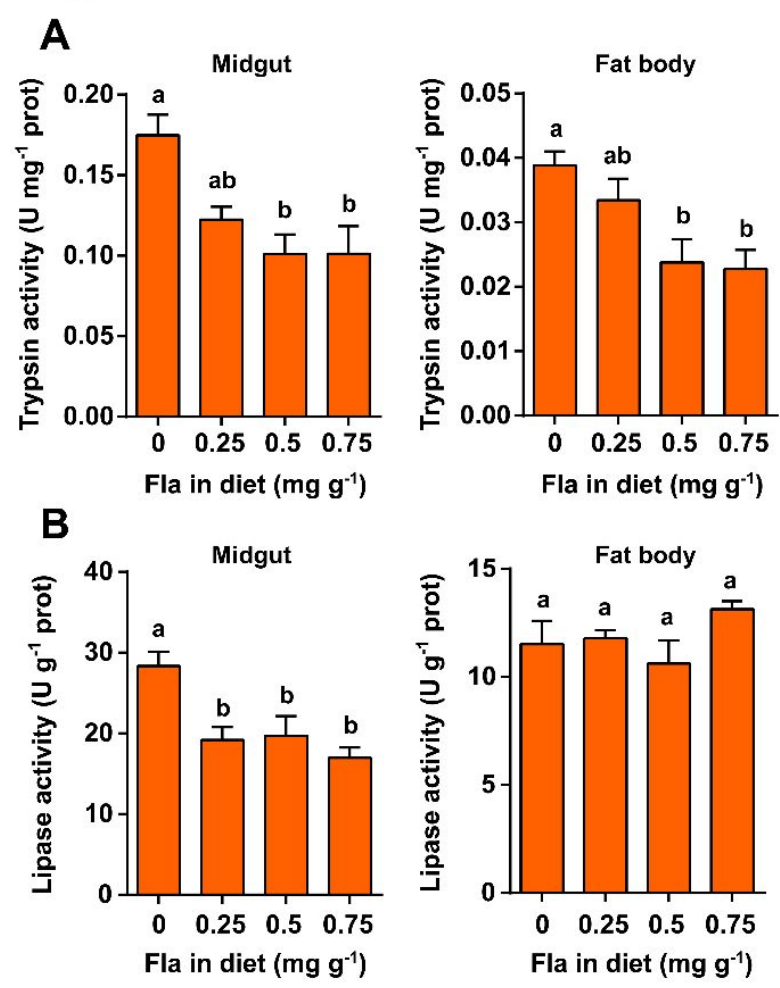

Figure S2. Activities of trypsin (A) and lipase (B) of flavone-treated S. litura larvae.

The $4^{\text {th }}$ instar larvae were fed on artificial diet containing different doses of flavone for $48 \mathrm{~h}$; then, the midguts and fat bodies were collected for enzymatic analyses. Results are expressed as means \pm SE of four biological replicates. Different lowercase letters above the bars indicate significant differences (one-way ANOVA followed by Tukey HSD multiple comparison test, $P<0.05)$. 
Fig. S3
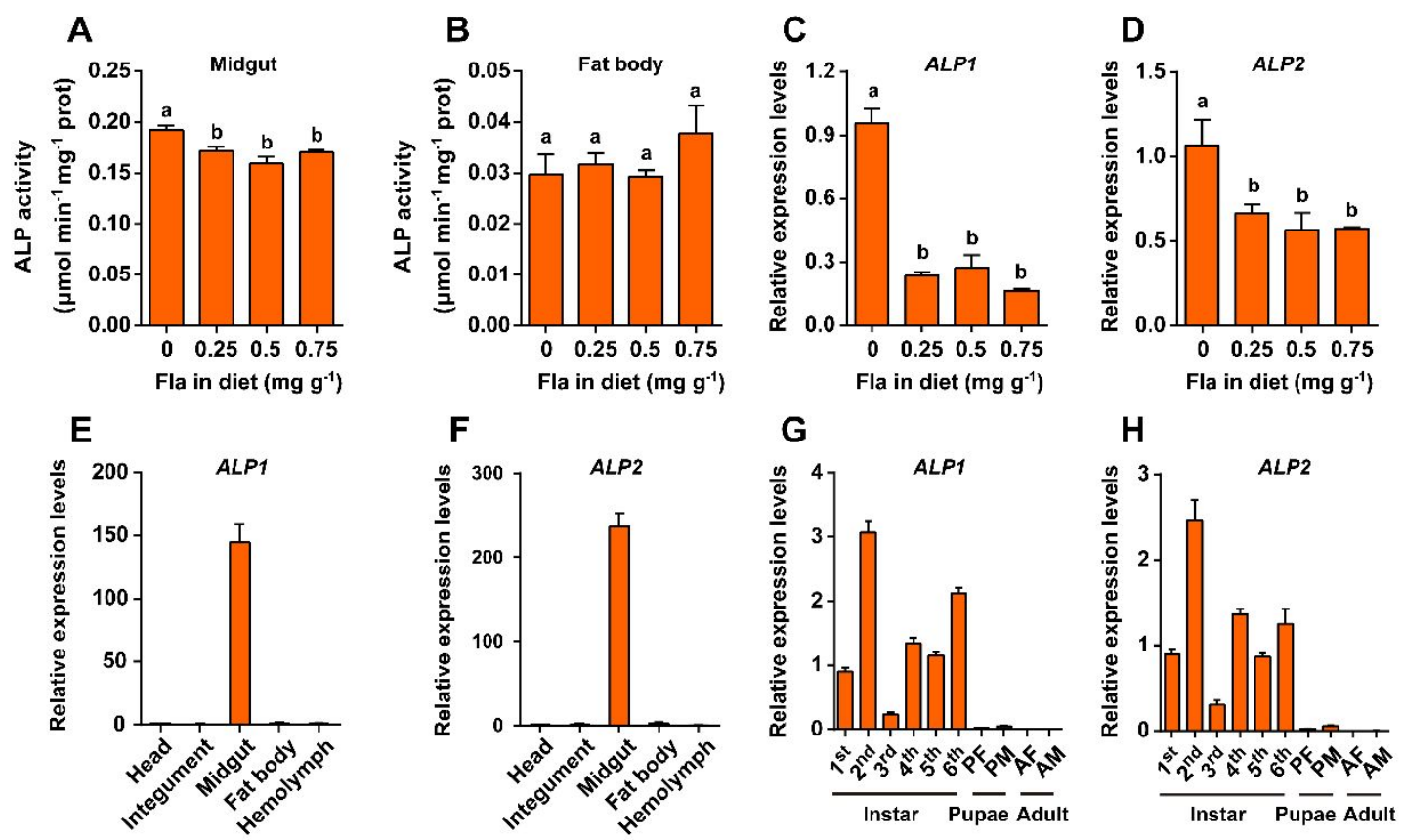

Figure S3. Effects of flavone exposure on alkaline phosphatase (ALP). The $4^{\text {th }}$ instar larvae were fed on artificial diet containing different doses of flavone for $48 \mathrm{~h}$. Activities of ALP in the midgut (A) and fat body (B) of flavone-treated S. litura larvae. Transcript levels of $A L P 1$ (C) and $A L P 2$ (D) in the larval midgut were determined by qRT-PCR. Tissue-specific expression of $A L P 1$ (E) and $A L P 2$ (F) determined by RTqPCR. Developmental-dependent expression patterns of $A L P 1(\mathrm{G})$ and $A L P 2(\mathrm{H})$ were determined by RT-qPCR. PF, pupae female; PM, pupae male; AF, adult female; MA, adult male. The mean expression in each developmental stage is represented as a fold change compared with the mean expression in the $1^{\text {st }}$ instar larvae, which has been ascribed an arbitrary value of 1 . Results are expressed as means \pm SE of four biological replicates. Different lowercase letters above the bars indicate significant differences (one-way ANOVA followed by Tukey HSD multiple comparison test, $P<0.05$ ). 
Table S1. Primers used in this study.

\begin{tabular}{|c|c|}
\hline Primer name & Primer sequence $\left(5^{\prime}-3^{\prime}\right)$ \\
\hline \multicolumn{2}{|l|}{ For qRT-PCR } \\
\hline$A L P 1-\mathrm{qF}$ & GGACGAGACGCTGGAGATGG \\
\hline$A L P 1-\mathrm{qR}$ & ACGTGGGAGTGATCCGCTGT \\
\hline$A L P 2-\mathrm{qF}$ & TGCCACTACTGAGCCCACGTT \\
\hline$A L P 2-\mathrm{qR}$ & CGACCACCTTCCACGAACAGA \\
\hline $\operatorname{CarE-\mathrm {qF}}$ & TACGGAAACCCAACGCCTGA \\
\hline CarE-qR & GGTGCCCGCTTTTAGCTCGT \\
\hline$C X E 4-\mathrm{qF}$ & TGGTTGGCTGGGGAGAAATG \\
\hline$C X E 4-\mathrm{qR}$ & AGCATTGCCTGGTGCGATTT \\
\hline$C X E 7-\mathrm{qF}$ & TGGGTGTTCACGGCTTCCTT \\
\hline$C X E 7-\mathrm{qR}$ & CTGCACCCAACGCAACAATG \\
\hline$C X E 8-\mathrm{qF}$ & CCGGTAATGCCGGTCTGAAA \\
\hline$C X E 8-\mathrm{qR}$ & CAACGACACGGATGCTGCAC \\
\hline CXE14-1-qF & CTGAAGTTGCGCCAGGCAAT \\
\hline CXE14-1-qR & TTGGGTTACCGCCAAAGCTG \\
\hline CXE14-2-qF & CCTTTGCCTCGGCACTGAAG \\
\hline CXE14-2-qR & ACCACCAAAGCTGGCGATGT \\
\hline CYP314A1-qF & CCTGGGAAGCGATTTGTGGA \\
\hline$C Y P 314 A 1-\mathrm{qR}$ & TAACCGCAGCGACACTGGAG \\
\hline$C Y P 321 A 7-\mathrm{qF}$ & GATGGAAGCTGATGCGACAGAA \\
\hline$C Y P 321 A 7-\mathrm{qR}$ & CCAGCATGGGTCTTTGGATTG \\
\hline$C Y P 321 A 8-\mathrm{qF}$ & CAACAGGACATGCATCGGTGA \\
\hline$C Y P 321 A 8-q R$ & TCGCAATTTCTCGGTCTCACG \\
\hline CYP321A10-qF & CAATCCCAAGACGACCGAAGG \\
\hline$C Y P 321 A 10-q R$ & CCAATGCCGAAGATAGCACCA \\
\hline CYP321A12-qF & GACCCTGAGCGGTTCTCCAA \\
\hline$C Y P 321 A 12-q R$ & ACTTGGCGTACCTGGCACCT \\
\hline$C Y P 321 B 1-q F$ & CTGGAGCATTTGGACCTTTG \\
\hline CYP321B1-qR & TCAGCTTGCCGCCGTATTTC \\
\hline$C Y P 333 A 6-q F$ & GCGACAAATCCGGAAAAACAA \\
\hline$C Y P 333 A 6-q R$ & CCTCAAATTAGCCGGGATGACA \\
\hline CYP6AE43-qF & TTCGGCACATTGCCCGTCCT \\
\hline CYP6AE43-qR & CGGTCGCCTGAACCGAAGAA \\
\hline
\end{tabular}




\begin{tabular}{|c|c|}
\hline Primer name & Primer sequence $\left(5^{\prime}-3^{\prime}\right)$ \\
\hline CYP6AE48-qF & AGCCGACACCAAGGAACGACT \\
\hline CYP6AE48-qR & ACGTCTCATACCCGGCAGCA \\
\hline CYP6B48-qF & GAGGTTTGGCAGACTCCAGTCAC \\
\hline$C Y P 6 B 48-q R$ & TCCCTCCTTTGGGTCCGATAATAAA \\
\hline CYP6B50-qF & TGGGCCAAGGAGTTGTCTTGGT \\
\hline CYP6B50-qR & TCGCGTGGGAGTGTACGTGA \\
\hline CYP9A9-qF & CCAATCCACCGAGACCCTCA \\
\hline CYP9A9-qR & GTCCGACACCGAAGGGCATA \\
\hline CYP9A39-qF & AACGGTTCGTTGGAAGGTA \\
\hline$C Y P 9 A 39-q R$ & TGGTGGTCAAGGAAGTGCT \\
\hline$G S T 1-q F$ & GACCAAATTCGTGGCAGCAGA \\
\hline$G S T 1-q R$ & TGTGTTGAACCAGGCCGTCA \\
\hline GST1-1-qF & GACCCTGACGTGGAGCGAGT \\
\hline$G S T 1-1-q R$ & GAGGGCGCAGTGGTCAGGTA \\
\hline$G S T-D 2-q F$ & CCCATGCTGTTTCGTGGTGA \\
\hline$G S T-D 2-q R$ & CCGTCCATCGAGGAAGGTGT \\
\hline GST-O2-qF & GATCTGGCCCTGGTTCGAAAG \\
\hline$G S T-O 2-q R$ & CTCCGCCACAACTGGGTCTT \\
\hline$G S T-S 1-q F$ & TGGACGACTGGCCGAAGTTT \\
\hline$G S T-S 1-q R$ & CGAGCGATGGCGATACTCTG \\
\hline$G S T-S 6-q F$ & ACAGTTGGCCAGACCCGAAA \\
\hline$G S T-S 6-q R$ & GGTGATGGCCAGGCTTTGAG \\
\hline$G S T-Z 2-q F$ & TTGCGTCGGAGACCAGCTCA \\
\hline$G S T-Z 2-q R$ & ACTGATGTCCAGCGCGTGTCT \\
\hline$U G T 33 B 13-q F$ & AGGAATTACCGGCGGATCTCA \\
\hline$U G T 33 B 13-q R$ & TGCGGTAGCAGTGAAGGTTGG \\
\hline$U G T 33 B 14-q F$ & GCACGACCAGCCTCAACCTC \\
\hline$U G T 33 B 14-q R$ & CGAGGTAGTCTGCCCACGACA \\
\hline$U G T 33 B 15-q F$ & CAAACGTCCAGCCTTCACTGC \\
\hline$U G T 33 B 15-q R$ & TTCGTCCTTGTCCCACTTCCA \\
\hline$U G T 33 J 2-q F$ & CACGGAGGAGCGAAACACCT \\
\hline$U G T 33 J 2-q R$ & GGCAATGGCAACGGCTAAAG \\
\hline$U G T 33 T 3-q F$ & AGTCCACAGACGAGGCCATCA \\
\hline$U G T 33 T 3-q R$ & CAGCGCTTCCATGTCCAGTCT \\
\hline
\end{tabular}




\begin{tabular}{|c|c|}
\hline Primer name & Primer sequence $\left(5^{\prime}-3^{\prime}\right)$ \\
\hline$U G T 33 V 3-q F$ & CGTGGATCCAGCATTGTTTCC \\
\hline$U G T 33 V 3-q R$ & CTGTTTTTCCCGGCAGTTCGT \\
\hline$U G T 40 F 4-q F$ & GGACAATGCCAAGAACGGTGT \\
\hline$U G T 40 F 4-q R$ & TCCTCCGAACACCTCCAACAA \\
\hline$U G T 40 M 2-q F$ & CGATCGCTTGACGAAACCTG \\
\hline$U G T 40 M 2-q R$ & TGGTACGTGGAGTGCTGGAGA \\
\hline$U G T 40 R 3-q F$ & TGCCCCTAAGTTTGCCACAGA \\
\hline$U G T 40 R 3-q R$ & TGGCCAGTCCTTGCTCTTCA \\
\hline$U G T 40 U 1-q F$ & GGCCAGCTCACCTCATCACC \\
\hline$U G T 40 U 1-q R$ & CGCTGCAACCGCTTCATTCT \\
\hline$U G T 41 B 1-q F$ & AACCGGCGCCGTTACCTAAG \\
\hline$U G T 41 B 1-q R$ & AGAGCCGATGAGCGCAGAAT \\
\hline$U G T 42 B 2-q F$ & TGGATCCCAAATTCAGGCAAA \\
\hline$U G T 42 B 2-q R$ & TGGCAGCGAATTCTGTCCAGT \\
\hline$U G T 42 C 2-q F$ & TGCCGCCACTTGGTGACTTA \\
\hline$U G T 42 C 2-q R$ & TCACGTACGTTTGGCGGGTA \\
\hline$U G T 46 A 4-q F$ & TGGGAAGGCCATTTCTTACGC \\
\hline$U G T 46 A 4-q R$ & ACAGCGGTGTCCAAGGGATCT \\
\hline$C n c C-q F$ & ACCGTCAGGGACAGGAAGCA \\
\hline$C n c C-q R$ & AGGTCGCGGAGGTTCTGGAAT \\
\hline$M a f K-q F$ & TCACCCGGGACCAGATAGTCA \\
\hline$M a f K-q R$ & TCCTTCTGCTCGATGCGTTTG \\
\hline Duox- $q F$ & AAGGCCCCTGGACATGGAAA \\
\hline Duox-qR & CCACCGCCTACCATCACTGC \\
\hline Nox4-qF & CAACGACGTGGCTGTGGATG \\
\hline Nox4-qR & TTATAGTGCGCCCCGGCATA \\
\hline Nox5-qF & AGGCATCCTGCTCCCCTCTC \\
\hline Nox5-qR & CGTAGTCTCCGGCGTGGAAG \\
\hline$E F-1 \alpha-q F$ & ACAGACAAGCCCCTGCGTCT \\
\hline$E F-1 \alpha-q R$ & TTGTCACCGGGTACGGCCTCTT \\
\hline \multicolumn{2}{|c|}{ For dsRNA synthesis } \\
\hline $\mathrm{CncC}-\mathrm{Fi}$ & ggatcctaatacgactcactatagggGCTGGACCTCAACACTGCACA \\
\hline$C n c C-R i$ & ggatcctaatacgactcactatagggTCGCGGAGGTTCTGGAATACA \\
\hline$G F P-F i$ & ggatcctaatacgactcactatagggCTGTCAGTGGAGAGGGTGAAGG \\
\hline
\end{tabular}


$\mathrm{F}$ : forward primer; R: reverse primer. Lowercase letters indicate the $\mathrm{T} 7$ promoter sequences. 
Table S2. Summary of the RNA-Seq data.

\begin{tabular}{lllllllll}
\hline Sample name & Raw reads & Clean reads & Clean bases (G) & Q20 (\%) & GC (\%) & Total reads & Total mapped & Mapping ratio (\%) \\
\hline CK1 & 89450080 & 88055956 & 13.21 & 96.87 & 48.13 & 88055956 & 79155979 & 89.89 \\
CK2 & 85661384 & 84599012 & 12.69 & 96.89 & 46.97 & 84599012 & 76362345 & 90.26 \\
CK3 & 84262526 & 82763366 & 12.41 & 97.01 & 47.43 & 82763366 & 74397510 & 89.89 \\
Fla1 & 82896076 & 81668020 & 12.25 & 97.12 & 46.4 & 81668020 & 73509526 & 90.01 \\
Fla2 & 92991298 & 91719518 & 13.76 & 96.92 & 45.67 & 91719518 & 82696646 & 90.16 \\
Fla3 & 87861634 & 86528188 & 12.98 & 97.03 & 46.29 & 86528188 & 78027273 & 90.18 \\
\hline
\end{tabular}


Table S3. Significantly enriched GO terms identified for the down-regulated DEGs in the midgut of $S$. litura exposed to Fla.

\begin{tabular}{lllll}
\hline GO_accession & Description & $\begin{array}{l}\text { Typ } \\
\text { e }\end{array}$ & $p$-value & FDR \\
\hline GO:0004252 & serine-type endopeptidase activity & MF & $4.274 \mathrm{E}-16$ & $1.9391 \mathrm{E}-12$ \\
GO:0008236 & serine-type peptidase activity & MF & $6.7268 \mathrm{E}-15$ & $1.0173 \mathrm{E}-11$ \\
GO:0017171 & serine hydrolase activity & MF & $6.7268 \mathrm{E}-15$ & $1.0173 \mathrm{E}-11$ \\
GO:0004175 & endopeptidase activity & MF & $2.3782 \mathrm{E}-12$ & $2.6795 \mathrm{E}-09$ \\
GO:0070011 & peptidase activity, acting on L-amino acid peptides & MF & $2.953 \mathrm{E}-12$ & $2.6795 \mathrm{E}-09$ \\
GO:0016787 & hydrolase activity & MF & $7.3569 \mathrm{E}-12$ & $5.563 \mathrm{E}-09$ \\
GO:0008233 & peptidase activity & MF & $1.5416 \mathrm{E}-11$ & $9.9918 \mathrm{E}-09$ \\
GO:0006508 & proteolysis & BP & $1.1709 \mathrm{E}-09$ & $6.6404 \mathrm{E}-07$ \\
GO:0003824 & catalytic activity & MF & $5.6335 \mathrm{E}-06$ & 0.0028399 \\
GO:0019538 & protein metabolic process & BP & 0.000041645 & 0.018895 \\
GO:1901565 & organonitrogen compound catabolic process & BP & 0.00004881 & 0.020132 \\
GO:0008745 & N-acetylmuramoyl-L-alanine amidase activity & MF & 0.000071951 & 0.027204 \\
GO:0008152 & metabolic process & BP & 0.000086486 & 0.030013 \\
GO:0006027 & glycosaminoglycan catabolic process & BP & 0.000099228 & 0.030013 \\
GO:0009253 & peptidoglycan catabolic process & BP & 0.000099228 & 0.030013 \\
GO:1901136 & carbohydrate derivative catabolic process & BP & 0.00015515 & 0.043995 \\
\hline
\end{tabular}

Table S4. List of the differential metabolites between the control and S. litura larvae treated with flavone. 